\title{
Total Hip Arthroplasty After Acetabular Fracture Is Associated With Lower Survivorship and More Complications
}

\author{
Zachary Morison MSc, Dirk Jan F. Moojen MD, PhD, Aaron Nauth MD, \\ Jeremy Hall MD, Michael D. McKee MD, James P. Waddell MD, \\ Emil H. Schemitsch MD
}

Published online: 3 September 2015

(C) The Association of Bone and Joint Surgeons (B) 2015

\begin{abstract}
Background Despite modern fracture management techniques allowing for near anatomic reduction of acetabular fractures, there continues to be a risk of posttraumatic arthritis and need for total hip arthroplasty (THA). Few well-controlled studies have compared THA after acetabular fractures with THAs performed for other indications in
\end{abstract}

Each author certifies that he or she, or a member of his or her immediate family, has no funding or commercial associations (eg, consultancies, stock ownership, equity interest, patent/licensing arrangements, etc) that might pose a conflict of interest in connection with the submitted article.

All ICMJE Conflict of Interest Forms for authors and Clinical Orthopaedics and Related Research ${ }^{\mathbb{R}}$ editors and board members are on file with the publication and can be viewed on request.

Clinical Orthopaedics and Related Research ${ }^{\mathbb{R}}$ neither advocates nor endorses the use of any treatment, drug, or device. Readers are encouraged to always seek additional information, including FDAapproval status, of any drug or device prior to clinical use. Each author certifies that his or her institution approved the human protocol for this investigation, that all investigations were conducted in conformity with ethical principles of research, and that informed consent for participation in the study was obtained.

This work was performed at St Michael's Hospital, Toronto, Ontario, Canada.

\section{Z. Morison ( $\square)$}

Li Ka Shing Institute, St Michael's Hospital, University of Toronto, West Basement B-114, 209 Victoria Street, Toronto, ON M5B 1W8, Canada

e-mail: morisonz@smh.ca; zmori086@gmail.com

D. J. F. Moojen

Department of Orthopaedics and Traumatology, Onze Lieve

Vrouwe Gasthuis, Amsterdam, The Netherlands

A. Nauth, J. Hall, M. D. McKee, J. P. Waddell, E. H. Schemitsch Division of Orthopaedic Surgery, Department of Surgery, St Michael's Hospital, Toronto, ON, Canada terms of survivorship or complications, and none, to our knowledge, present 10 -year survivorship data in this setting.

Questions/purposes (1) How does the 10-year survival of THA compare between those patients who underwent THA after an acetabular fracture and those who underwent THA for primary arthritis or avascular necrosis (AVN)? (2) Is there an increased risk of serious complications like infection, dislocation, and aseptic loosening as well as heterotopic ossification associated with a THA performed after a previous acetabular fracture?

Methods This retrospective case-control study compared findings of patients who underwent THA after acetabular fracture versus a matched cohort of patients who had received a primary THA for primary osteoarthritis or AVN. Between 1987 and 2011, we performed 95 THAs after acetabular fracture; of those, 74 (78\%) met inclusion criteria and had documented followup beyond 2 years in our institutional registry. We selected 74 matched patients based on an algorithm that matched patients based on preoperative diagnosis, date of operation, age, gender, and type of prosthesis. During this time, we performed approximately 8000 THAs that were potentially available for matching based on complete followup beyond 2 years. We compared cases and control subjects using the KaplanMeier survivorship estimator as well as a comparison of the proportions in each group that developed major complications (including infection, dislocation, loosening, and heterotopic ossification) based a retrospective chart review. Results The 10-year survivorship after THA was lower in patients with a previous acetabular fracture than in the matched cohort $(70 \%, 95 \%$ confidence interval [CI], 64\%$78 \%$, versus $90 \%, 95 \%$ CI, 86-95\%; $p<0.001)$. There was no difference in the 10-year survival rate for those patients whose acetabular fracture was initially treated 
conservatively and those treated by open reduction and internal fixation. Patients with previous acetabular fracture had a higher likelihood of developing infection (7\% [five of 74] versus $0 \%$ [zero of 74]; odds ratio [OR], 11.79; $\mathrm{p}=$ 0.028 ), dislocation (11\% [eight of 74 ] versus $3 \%$ [two of 74]; OR, 4.36; $\mathrm{p}=0.048$ ), or heterotopic ossification ( $43 \%$ [32 of 74] versus $16 \%$ [12 of 74]; OR, 3.93; $p<0.001$ ).

Conclusions In this case-control study, patients with a prior acetabular fracture had markedly inferior 10-year survivorship and more frequent serious complications when compared with patients undergoing THA for primary osteoarthritis or AVN. Given these findings, management of these complex cases should be in highly specialized units where the expertise of arthroplasty and trauma reconstruction is available.

Level of Evidence Level III, therapeutic study.

\section{Introduction}

Acetabular fractures are complex intraarticular injuries that occur in a bimodal distribution, typically in younger patients involved in high-energy blunt trauma and in older patients with low-energy falls in the setting of osteoporosis. Although modern fracture management techniques allow for near anatomic reduction of these fractures, there continues to be a risk of posttraumatic arthritis $[10,13]$. In a meta-analysis conducted by Giannoudis et al. [6] investigating the operative treatment for displaced acetabular fractures found that despite satisfactory reduction $(\leq 2 \mathrm{~mm})$ with open reduction and internal fixation (ORIF), the incidence of osteoarthritis (OA) was $13 \%$ (76 of 577 patients). However, if the reduction was not satisfactory $(>2 \mathrm{~mm})$, the incidence was markedly increased to $44 \%$ (47 of 108 patients) [6]. Management of posttraumatic arthritis of the hip after acetabular fractures with THA has demonstrated a propensity for revision as compared with THA for nontraumatic OA [8, 13, 15]. This increased failure rate was typically realized in cemented acetabular components and was attributable to factors such as the relatively younger population, abnormal anatomy after trauma, sclerotic bone bed, loss of acetabular bone stock, and complications involving previous hardware. However, cementless acetabular fixation and development in implant designs have improved survivorship [1]. Many factors play a role in the survivorship of THA after acetabular fracture including age, management of the initial fracture, fracture pattern, and the amount of displacement.

Although some studies have evaluated patients undergoing THA after acetabular fracture at long-term followup [5, 14], and others have compared THAs performed after acetabular fractures with THAs performed for other indications [11], few have reported 10-year survivorship data in a well-controlled study. The aim of this retrospective matched cohort study is to investigate the long-term survival and clinical and radiographic results in patients who have undergone THA after an acetabular fracture as compared with patients who underwent THA for primary OA or avascular necrosis (AVN).

We therefore asked: (1) How does the 10-year survival of THA compare between those patients who underwent THA after an acetabular fracture and those who underwent THA for primary arthritis or AVN? (2) Is there an increased risk of serious complications like infection, dislocation, and aseptic loosening as well as heterotopic ossification associated with a THA performed after a previous acetabular fracture?

\section{Patients and Methods}

This retrospective case-control study compared findings of patients who underwent THA after acetabular fracture versus a matched cohort of patients who had received a primary THA for primary arthritis or AVN. Institutional review board approval was obtained and all patients were identified from existing trauma and arthroplasty databases. Between January 1, 1987, and March 31, 2011, approximately 650 patients were treated with acetabular fractures at a Level I trauma center. Of those patients, 95 were subsequently treated with THA at a median of 4 years (range, 1-26 years). Nine patients (9\%) had died, 12 (13\%) were lost to followup, and 74 patients (74 hips [78\%]) were available for followup at a minimum of 2 years (median, 8 years; range, 2-23 years). Thus, these 74 patients met the inclusion criteria, which included evidence of posttraumatic OA or AVN, uncemented acetabular components, and minimum followup for case matching. During the same study period, we performed approximately 8000 THAs for primary OA or AVN that were potentially eligible for case matching because they were available for followup at a minimum of 2 years (median, 10 years; range, 2-24 years). Once the subjects were identified, charts were retrospectively reviewed for demographic data, type of fracture based on the classification of Letournel [9], mechanism of trauma (eg, fall, motor vehicle accident), initial treatment of the fracture, time between fracture and THA, type of prostheses, complications, followup time, and revision rate. A matched cohort was then chosen from patients with primary arthritis or AVN who underwent primary THA by surgeons at the same institution during the same period. One control patient was selected for each study patient and was matched for preoperative diagnosis, date of operation (within 1 year), age, gender, and type of prosthesis to control for their confounding effects (Table 1). Minimum followup for inclusion was set at 2 years.

All THAs were performed using a posterior surgical approach. The acetabulum was prepared as for routine 
Table 1. Demographic data for the study group and control group

\begin{tabular}{|c|c|c|c|}
\hline Demographics & $\begin{array}{l}\text { Study } \\
\text { group }\end{array}$ & $\begin{array}{l}\text { Control } \\
\text { group }\end{array}$ & $\mathrm{p}$ value \\
\hline Number of patients & 74 & 74 & \\
\hline $\begin{array}{l}\text { Mean age (years; } \\
\text { range) (SD) }\end{array}$ & $51(25-75)(12)$ & $52(30-81)(12)$ & 0.557 \\
\hline Male:female & $50: 24$ & $50: 24$ & \\
\hline $\begin{array}{l}\text { Median followup } \\
\text { (years; range) }\end{array}$ & $8(2-23)$ & $10(2-24)$ & 0.162 \\
\hline \multicolumn{4}{|l|}{ Preoperative diagnosis } \\
\hline Osteoarthritis & 60 & 60 & \\
\hline Avascular necrosis & 14 & 14 & \\
\hline \multicolumn{4}{|l|}{ Implant design (stems) } \\
\hline Synergy & 34 & 34 & \\
\hline Echelon & 16 & 16 & \\
\hline Spectron & 4 & 4 & \\
\hline St. Michael's & 20 & 20 & \\
\hline \multicolumn{4}{|l|}{ Implant design (cups) } \\
\hline Reflection Cups & 47 & 50 & \\
\hline R3 & 3 & 4 & \\
\hline St. Michael's & 20 & 20 & \\
\hline TM Cup & 4 & - & \\
\hline \multicolumn{4}{|l|}{ Implant fixation } \\
\hline Hybrid & 4 & 4 & \\
\hline Uncemented & 70 & 70 & \\
\hline
\end{tabular}

primary THAs and hardware from previous surgeries was not routinely removed unless it was interfering with reaming and fitting of the cup. Seventy of the components inserted were cementless, and four had hybrid fixation with cementless cups and cemented femoral components. The patients were matched for date of surgery to account for changes in technique or technology that occurred over the duration of this study. The patients were matched according to stem and cup design. The principle stem design (34 of 74 [46\%]) was a tapered, proximally coated stem (Synergy; Smith \& Nephew, Memphis, TN, USA); other designs were the cylindrical proximally coated stem (Echelon; Smith \& Nephew) and the St Michael's Stem, which had a madreporic beaded coating proximally and is no longer available (Howmedica, Mahwah, NJ, USA). A cemented stem (Spectron; Smith \& Nephew) was used in four (four of 74 [5\%]) of patients. Acetabular cup designs were primarily the porous-coated cementless acetabular shells (Reflection and R3; Smith \& Nephew) and the St Michael's Cup (Howmedica). All implants used cobaltchromium femoral heads and polyethylene liners.

Postoperative care for all patients included first-generation cephalosporins for 24 to 48 hours postoperatively. All patients received heterotopic ossification prophylactic treatment with $25 \mathrm{mg}$ indomethacin three times daily for 10 days. Patients were restricted to partial weightbearing for 6 weeks postoperatively and rehabilitation included ROM, strength, and balance exercises with the supervision of a physiotherapist. The recommended course of physiotherapy for these patients was twice a week for 3 months.

The primary outcomes were revision and incidence of complications. Secondary outcomes were radiographic signs of heterotopic ossification or implant loosening. For this, radiographs obtained at the last followup visit from each patient were evaluated for heterotopic ossification using the Brooker classification [3], radiolucencies around the cup according to the DeLee and Charnley zones [4], and radiolucencies about the femoral stem using Gruen zones [7]. For this study, we defined acetabular loosening as circumferential radiolucent lines $>2 \mathrm{~mm}$ and/or evidence of migration. All radiographs were evaluated by a single, blinded observer (DJM). Radiographs were available in 70 acetabular fracture and 73 control patients.

Nonparametric tests including Fisher's exact test, Kaplan-Meier survival analysis, Mann-Whitney, and analysis of variance tests were used to compare the results of patients who had undergone THA after prior acetabular fracture with those of patients who had primary THA for primary arthritis or AVN arthritis at our institution. The endpoint for the survival analysis was revision for any reason. Statistical differences were considered significant at $\mathrm{p}<0.05$. All patients who were treated for acetabular fracture (both operatively and nonoperatively) and subsequently underwent uncemented THA for posttraumatic arthritis or AVN with minimum followup of 2 years were eligible for inclusion in the study. Thus, the study size was determined by the number of patients who were treated at our institution during the study period.

The cohort of acetabular fracture patients included 50 men and 24 women with a mean age of 52 years (SD, 12 years) and median followup of 8 years (range, 2-23 years). Patients with a previous acetabular fracture required a THA at a median time of 4 years (range, 1-24 years). Each study patient was matched to a control patient. There were no differences between the cohorts for preoperative data (Table 1). The majority of patients (60 of 74 [81\%]) had a preoperative diagnosis of osteoarthritis and the remaining patients (14 of 74 [19\%]) had AVN. A subanalysis was conducted to determine the difference in survivorship between patients younger than 60 years and those 60 years and older at the time of their THA; $34 \%$ (26 of 74) of the cohort was $\geq 60$ years old.

The majority of acetabular fractures were treated by ORIF (58 of 74 [78\%]), whereas 16 of $74(22 \%)$ were treated nonoperatively. The most frequent type of fracture involved the posterior wall, accounting for $31 \%$ of all injuries. Fractures involving both columns were seen in $16 \%$, whereas other fracture types were less common and 
Table 2. Acetabular fracture treatment and fracture pattern

\begin{tabular}{ll}
\hline Treatment or fracture pattern & Study group, number \\
\hline Treatment for acetabular fracture & $58(78 \%)$ \\
ORIF & $16(22 \%)$ \\
Conservative & \\
Elementary & $2(3 \%)$ \\
Anterior column & $5(7 \%)$ \\
Posterior column & $23(31 \%)$ \\
Posterior wall & $5(7 \%)$ \\
Transverse & 35 \\
Total & \\
Associated & $4(5 \%)$ \\
Anterior + posterior hemitransverse & $12(16 \%)$ \\
Both columns & $8(11 \%)$ \\
Posterior column + posterior wall & $7(9 \%)$ \\
Transverse + posterior wall & $8(11 \%)$ \\
T-type & 39 \\
Total &
\end{tabular}

ORIF $=$ open reduction and internal fixation.

were seen in less than $10 \%$ of patients (Table 2). Overall, $49 \%$ of the fractures were classified as elementary fracture patterns and $51 \%$ were associated fractures.

\section{Results}

The 10-year survivorship after THA was lower in patients with a previous acetabular fracture than in the matched cohort (70\%, 95\% confidence interval [CI], 64\%-78\% versus $90 \%$, 95\% CI, 86\%-95\%; p < 0.001) (Fig. 1). Younger patients $(<$ 60 years) had worse THA survivorship after acetabular fractures than did older patients $(60 \%, 95 \%$ CI, 51\%-69\% versus 83\%, 95\% CI, 72\%-94\%; p < 0.038) (Fig. 2), and both had inferior survivorship to the matched cohort (92\%, CI, 87\%$97 \%$ and $96 \%$ CI, 92\%-99\%; $<<0.001$ ). The 10 -year survival for THA after a simple acetabular fracture was $83 \%(95 \% \mathrm{CI}$, $77 \%-89 \%)$ as compared with $60 \%$ (95\% CI, 52\%-68\%; p = 0.032 ) for associated fractures.

The time from the initial THA to the revision in patients with previous acetabular fracture (8 years; SD, 5 years) was shorter than in the matched cohort (13 years; SD, 6 years; $p$ $=0.015)$. With the numbers available, there was no difference in subsequent revisions for those patients who had their initial acetabular fracture treated by ORIF (21 of 59 [36\%]) compared with those whose acetabular fracture was treated conservatively (three of $18[17 \%])(p=0.15)$. The primary cause for revision in both cohorts was loosening of the acetabular components. In patients with previous acetabular fracture, 13 patients $(20 \%)$ were revised for loose acetabular component, six patients for wear and joint instability (8\%), two for infection, and one each for femur fracture, loose femoral component, and recurrent dislocation. Revisions for the matched cohort included 11 patients for cup loosening and one patient for recurrent dislocations.

Patients with previous acetabular fracture were more likely to develop serious complications. including infection, dislocation, and acetabular loosening; they also were more likely to develop heterotopic ossification. Our findings demonstrated that patients with previous acetabular fracture had a higher likelihood of developing infection than did patients whose indication for THA was nontraumatic arthritis (7\% [five of 74] versus 0\% [zero of 74]; odds ratio [OR], 11.79; $\mathrm{p}=0.028)$. Similarly, patients with previous acetabular fracture also had a higher likelihood of dislocation (11\% [eight of 74] versus 3\% [two of 74]; OR, $4.36 ; \mathrm{p}=0.048)$ and onset of heterotopic ossification $(43 \%$ [32 of 74] versus $16 \%$ [12 of 74]; OR, 3.93; p < 0.001) compared with the control group. Ten patients in the acetabular fracture group already had a sciatic nerve lesion before the THA; one additional patient had a lesion after the THA. No patients in the control group had a sciatic nerve lesion.

Of the 51 patients in the acetabular fracture group, who did not have a revision, six had no radiographs available, 46 had well-fixed components, and none had cup loosening. Of the 62 control patients without revision, three had no radiographs available, 59 had well-fixed components, and none had cup loosening. Heterotopic ossification was seen in 32 patients $(45 \%)$ with a previous acetabular fracture. Of those, 30 patients were classified as Brooker Class II or less and only two were Class IV. Comparatively, the matched cohort had a total of 12 patients $(16 \%)$ with heterotopic ossification and none were Class IV.

\section{Discussion}

Acetabular fractures represent complex injuries of the hip and are associated with high morbidity and the development of posttraumatic OA can occur even after anatomical reconstruction. Posttraumatic arthritis of the hip may develop not only as a result of residual articular incongruity, but also as the result of the damage to the articular cartilage at the time of the injury often resulting in a painful joint and impairment of function. In these cases, THA is often recommended to restore function of the hip. In the existing body of literature, the mid- and long-term results of THA after acetabular fracture are conflicting. Weber et al. [15] and Romness and Lewallen [13] reported unsatisfactory results, especially for aseptic acetabular loosening over the long term, with rates approaching 50\%. However, Bellabarba et al. [1] and Ranawat et al. [12] reported satisfactory results with a midterm survival rate of $97 \%$ for acetabular 
Fig. 1 This figure displays the Kaplan-Meier survival comparing the 10-year survival of the two cohorts of patients.

Fig. 2 This figure displays the Kaplan-Meier survival comparing the 10-year survival of the experimental cohort stratified by age.
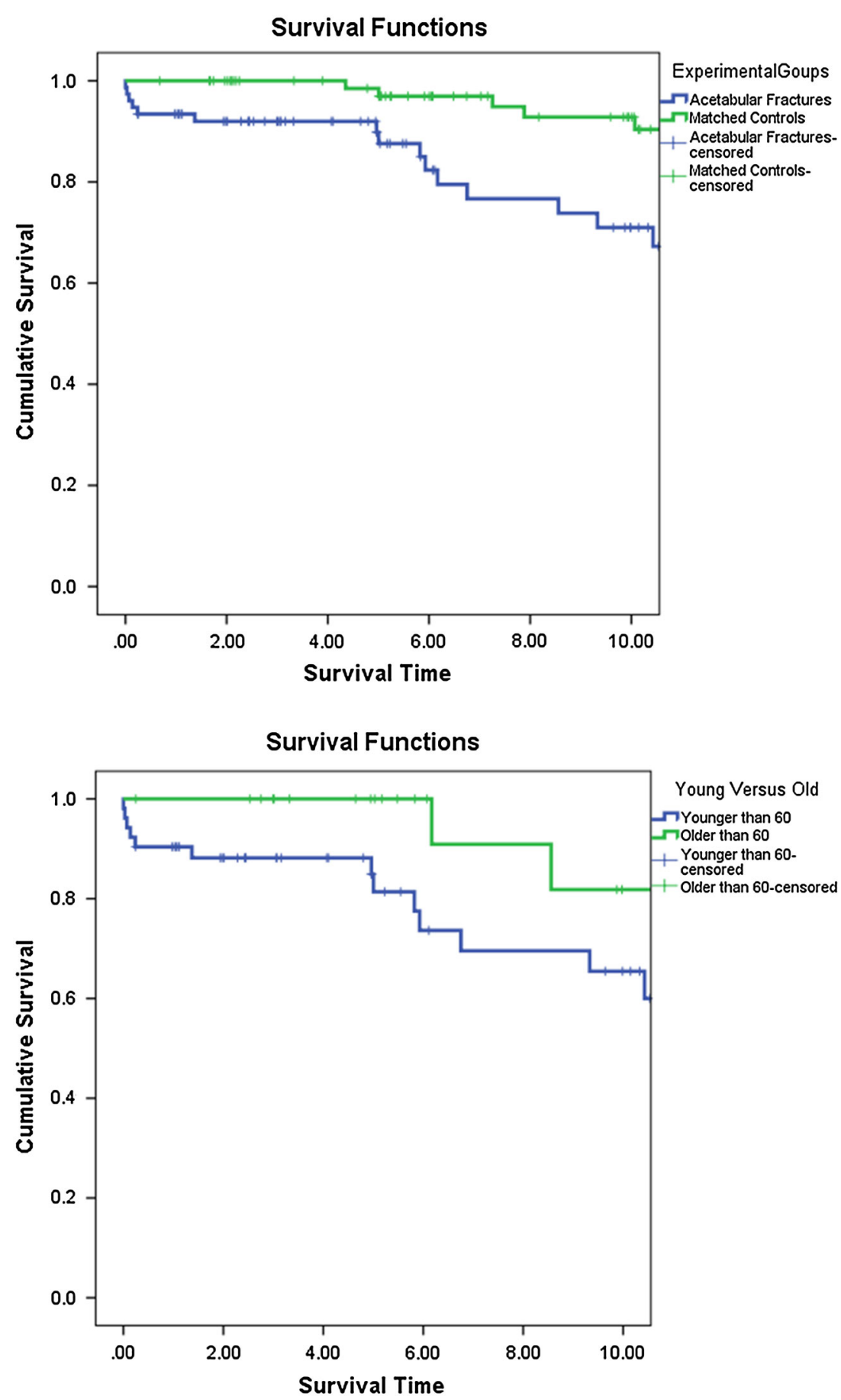

components. Few well-controlled studies have compared THA after acetabular fractures with THAs performed for other indications in terms of survivorship or complications, and none, to our knowledge, present 10-year survivorship data in this setting. The current study found that patients with a prior acetabular fracture had markedly inferior 10year survivorship and experienced more frequent serious complications when compared with patients undergoing THA for nontraumatic causes.
There are a number of limitations to the current work. In particular, the absence of a validated outcome score such as the Harris hip score or WOMAC limits our ability to measure the functional outcomes of these patients. During the period of this study, our institution used a scoring instrument that was not validated. Additionally, there are inherent weaknesses of using a retrospective study design such as the potential for selection bias. As a Level I trauma center and tertiary care academic hospital, there is a risk that acetabular fracture 
patients seen at our institution present with above average Injury Severity Scores (ISS). ISS were not available for many of the patients screened for this study and as a result, we included all patients with acetabular fractures with the intent to maximize the external validity of our findings. Moreover, it can be challenging to control for confounding variables in retrospective studies; however, by using a case-control design, we were able to control for many of the baseline characteristics of both groups. Furthermore, a retrospective case-control study is beneficial in this circumstance given the long followup after the initial trauma to the acetabulum and the onset of posttraumatic OA. Prospective study designs are inefficient for investigating diseases with long latency periods such as $\mathrm{OA}$ and are vulnerable to a high loss to followup. Conversely, given the large numbers of patients undergoing THA over the course of this study period, it was not feasible to determine the exact loss to followup. As a result, it is not possible to determine if there was differential loss to followup between the two cohorts. There was, however, no difference in the duration of followup between cohorts.

The findings from the current study are in agreement with other published studies that have shown there is an increased revision rate in patients undergoing THA after an acetabular fracture. This study found that THAs after a previous acetabular fracture have worse outcome survivorship in younger patients $(<60$ years) and revision THA occurred on average 5 years earlier than those without a prior acetabular fracture. Romness and Lewallen reported revisions in $17 \%$ of patients younger than 60 years of age with delayed joint replacement and $8 \%$ in patients older than 60 years old [13]. Similarly, Berry and Halasy [2] found that age younger than 50 years is a risk factor for aseptic loosening after a 10-year followup. This increased failure rate is conceivably attributable to factors such as the relatively younger population, abnormal anatomy after trauma, sclerotic bone bed, loss of acetabular bone stock, and complications involving previous hardware.

Infection is a recurrent problem in this patient population as a result of multiple prior surgeries and retained hardware. The risk of infection in THA after acetabular fractures has been reported to be higher than conventional hip arthroplasty, especially in patients with multiple prior surgeries and retained hardware from previous acetabular reconstruction. This study also found that there were more cases of infection in THA after a previous acetabular fracture. The incidence of infection was $6 \%$ (five patients) with two patients requiring revision as a result of deep infections and three superficial infections revolved after antibiotics. Compared with the other series, our postoperative infection rate of $6 \%$ was higher: Weber et al. [15] reported no infections, Berry and Halasy [2] reported one unconfirmed superficial infection, and Bellabarba et al. [1] reported no deep infections; however, that series described one case with multiple débridements for infected trochanteric hardware. Postoperative dislocations are another common postoperative complication seen in patients undergoing THA. In a recent systematic review, Makridis et al. [11] found that the dislocations occur in $4.4 \%$ of patients undergoing THA after a previous acetabular fracture as compared with a range of $0.2 \%$ to $7 \%$ seen in conventional primary THA. Our study demonstrated a higher incidence of dislocation in THA secondary to acetabular fractures compared with the cohorts of Weber et al. [15] (3\%) and Bellabarba et al. [1] (0\%), which may be attributable to a more common use of $20^{\circ}$ elevated liners in their cohorts. Lastly, the onset of heterotopic ossification in our series was higher in posttraumatic patients than in the control group. However, the formation of ectopic bone did not require any intervention in any of the patients after the THA. In a recent meta-analysis of THA after previous acetabular fracture, the rate of heterotopic ossification was $30 \%$. This is compared with highly variable rates of heterotopic ossification ranging from $5 \%$ to $90 \%$ in conventional primary THA [11].

Patients with a prior acetabular fracture who underwent THA had inferior 10-year survivorship to a matched cohort of patients undergoing THA who did not have a prior acetabulum fracture; the patients with prior acetabular fractures also underwent revision THA approximately 5 years earlier than those without a prior acetabular fracture. Additionally, this case-control study substantiates a higher incidence of complication in patients who have undergone THA after an acetabular fracture. Given these findings, management of these complex cases should be in highly specialized units where the expertise of arthroplasty and trauma reconstruction is available.

Acknowledgments We thank Kerry Ann Griffith-Cunningham and Jane Morton for their contributions toward the data collection necessary for this study.

\section{References}

1. Bellabarba C, Berger RA, Bentley CD, Quigley LR, Jacobs JJ, Rosenberg AG, Sheinkop MB, Galante JO. Cementless acetabular reconstruction after acetabular fracture. J Bone Joint Surg Am. 2001;83:868-876.

2. Berry DJ, Halasy M. Uncemented acetabular components for arthritis after acetabular fracture. Clin Orthop Relat Res. 2002;405:164-167.

3. Brooker AF, Bowerman JW, Robinson RA, Riley LH Jr. Ectopic ossification following total hip replacement. Incidence and a method of classification. J Bone Joint Surg Am. 1973;55:16291632.

4. DeLee JG, Charnley J. Radiological demarcation of cemented sockets in total hip replacement. Clin Orthop Relat Res. 1976;121:20-32.

5. Dunet B, Tournier C, Billaud A, Lavoinne N, Fabre T, Durandeau A. Acetabular fracture: long-term follow-up and factors associated with secondary implantation of total hip arthroplasty. Orthop Traumatol Surg Res. 2013;99:281-290. 
6. Giannoudis PV, Grotz MR, Papakostidis C, Dinopoulos H. Operative treatment of displaced fractures of the acetabulum. A meta-analysis. J Bone Joint Surg Br. 2005;87:2-9.

7. Gruen TA, McNiece GM, Amstutz HC. 'Modes of failure' of cemented stem-type femoral components: a radiographic analysis of loosening. Clin Orthop Relat Res. 1979;141:17-27.

8. Huo MH, Solberg BD, Zatorski LE, Keggi KJ. Total hip replacements done without cement after acetabular fractures: a 4- to 8-year follow-up study. J Arthroplasty. 1999;14:827-831.

9. Letournel E. Acetabulum fractures: classification and management. Clin Orthop Relat Res. 1980;151:81-106.

10. Letournel E. Fractures of the Acetabulum. Vol 2. 2nd ed. New York, NY, USA: Springer; 1993.

11. Makridis KG, Obakponovwe O, Bobak P, Giannoudis PV. Total hip arthroplasty after acetabular fracture: incidence of compli- cations, reoperation rates and functional outcomes: evidence today. J Arthroplasty. 2014;29:1983-1990.

12. Ranawat A, Zelken J, Helfet D, Buly R. Total hip arthroplasty for posttraumatic arthritis after acetabular fracture. J Arthroplasty. 2009;24:759-767.

13. Romness DW, Lewallen DG. Total hip arthroplasty after fracture of the acetabulum. Long-term results. J Bone Joint Surg Br. 1990;72:761-764.

14. von Roth P, Abdel MP, Harmsen WS, Berry DJ. Total hip arthroplasty after operatively treated acetabular fracture. J Bone Joint Surg Am. 2015;97:288-291.

15. Weber M, Berry DJ, Harmsen WS. Total hip arthroplasty after operative treatment of an acetabular fracture. J Bone Joint Surg Am. 1998;80:1295-1305. 